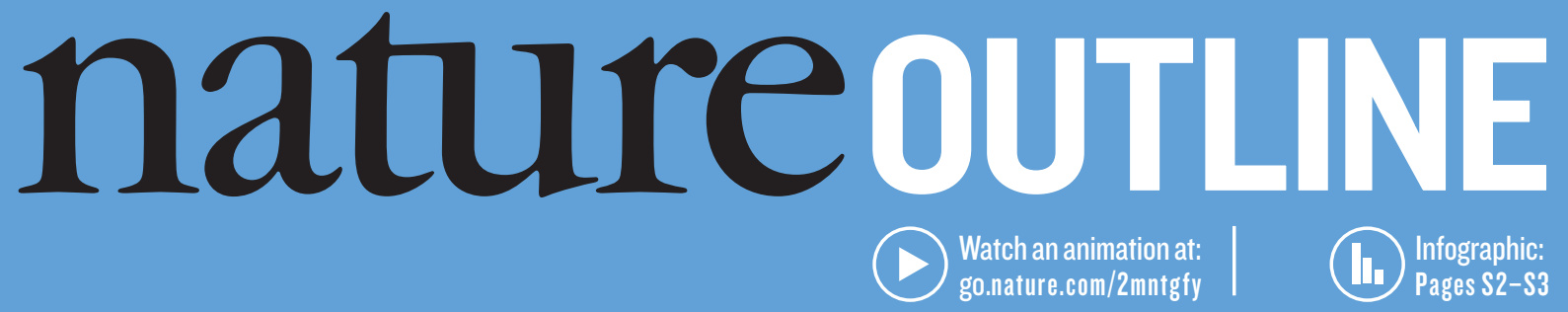

\title{
QUANTUM COMPUTING: TOWARDS REALITY
}

\section{The concept of computers that harness the laws of quantum mechanics has transformed our thinking about how information can be processed. Now the environment exists to make prototype devices a reality.}

\section{BY ANDREAS TRABESINGER}

ow fancy do our computers need to be? Nowadays, when artificial intelligence is in our homes and not just on the television, it might seem that computers can solve almost every problem. But in reality, progress in numerous areas of science and technology is limited because current computers are too inefficient. Even the most powerful supercomputers cannot accurately calculate the properties of novel materials, chemical structures or biological systems when there are too many interacting elements.

A new type of computing is needed, one that can take advantage of the very complexity that computers are trying to penetrate. Enter quantum computers, which were first mooted in the 1980s, when it was suggested that it might be possible to construct computers based on the laws of quantum physics instead of on classical physics. But such an approach requires assembling and controlling quantum systems - an exceedingly difficult task. Typically, quantum phenomena appear only at the level of the fundamental constituents of matter, such as atoms and electrons.

But once quantum systems are made accessible, they will offer a wealth of possibilities. To describe a general quantum state of just a few hundred particles, the number of variables needed exceeds the number of atoms in the Universe. No conventional computer will ever have the memory to store such an amount of information. But if the richness of quantum states is used to encode information, a whole new world of computational possibilities opens up. These go beyond simulating the behaviour of materials and molecules, to include fast searching through large databases and fundamentally novel approaches to cryptography.

The past two decades have seen steady progress in isolating, manipulating and measuring the elements that might form the

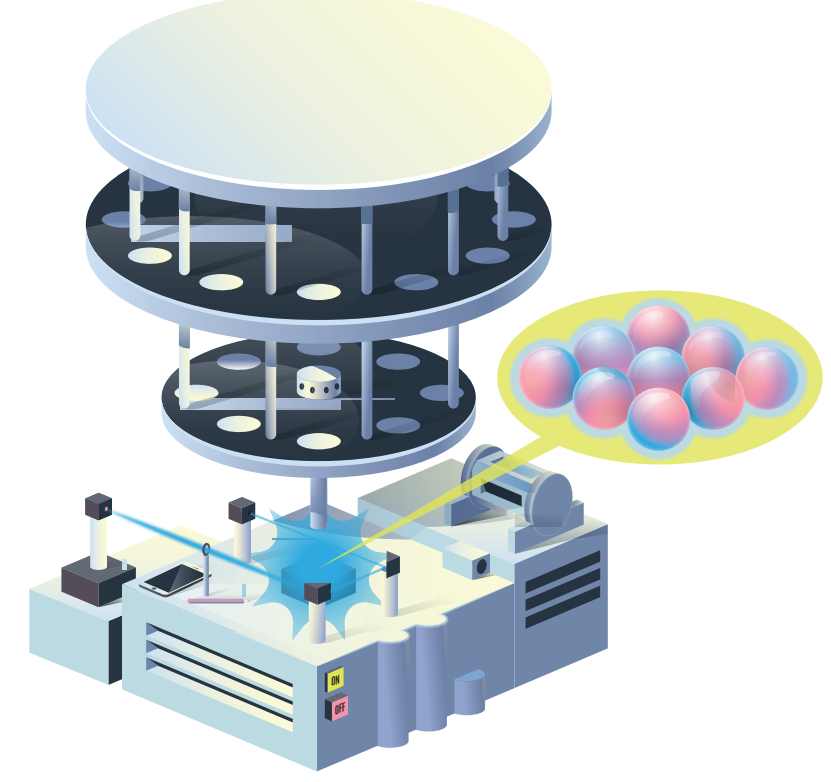

Much of quantum-computer architecture is devoted to keeping qubits in a delicate state of superposition.

basis of a quantum computer, be they single quantum entities such as atoms, electrons or photons, or artificial systems that display quantum-mechanical behaviour, such as semiconductor structures or miniature electronic circuits. Researchers now have proof of principle that quantum computers can work, but the tremendous scale of the challenges ahead is also clear.

Physicists, mathematicians, computer scientists and engineers at universities around the world are wrestling with the issue of how to construct and operate a quantum computer that is sufficiently large to beat classical computers at certain tasks. Commercial companies are getting involved, too. Just what a quantum computer will eventually look like, which quantum systems it will use, and which problems it will solve remain open questions. And these are fuelling exciting research.

Nature is pleased to acknowledge the financial support of Nokia Bell Labs in producing this Outline. As always, Nature retains sole responsibility for all editorial content.

Andreas Trabesinger is a science writer based in Switzerland. 